\title{
Patientensicherheit auf allen Stufen des Medikationsprozesses
}

\author{
Susanna Kussmann, Patrik Muff, Peter Wiedemeier, Laurie Bochatay, Johnny Beney, Carla Meyer-Massetti
}

GSASA, Schweizerischer Verein der Amts- und Spitalapotheker

Unerwünschte Arzneimittelwirkungen und Medikationsfehler gehören zu den grössten Risiken, welche die Patientensicherheit im Spital beeinträchtigen. Mindestens 7,5\% der Patienten in der Schweiz sind während ihres Spitalaufenthalts davon betroffen. Es wird geschätzt, dass etwa die Hälfte dieser Komplikationen vermeidbar wäre.

Die Medikationssicherheit muss durch Massnahmen im gesamten Medikationsprozess optimiert werden. Deshalb empfehlen internationale, evidenzbasierte Strategien die Stärkung der interdisziplinären Zusammenarbeit, die Verbesserung der Kommunikation zwischen allen in die medizinische Betreuung involvierten Personen und dem Patienten, die Standardisierung der Prozesse, die Fokussierung auf Hochrisiko-Medikamente sowie die Erhöhung des Informatisierungsgrades (elektronische Verordnung, ePatientendossier usw.).

Im Folgenden werden exemplarisch vier zentrale Bereiche angesprochen, in denen sich die GSASA resp. die Schweizer Spitalapotheker täglich für die Patientensicherheit einsetzen.

\section{Sicherheit durch Stärkung der interdisziplinären Zusammenarbeit}

Das Ziel, das richtige Medikament für die richtige Person in der richtigen Dosierung zum richtigen Zeitpunkt richtig anzuwenden (5R-Regel), sollte für jeden Patienten erreicht werden. Diesbezüglich können klinische Pharmazeuten als Arzneimittel-Fachspezialisten und Experten für den Medikationsprozess das medizinisch-pflegerische Team nutzbringend ergänzen [1]*. Ihre Interventionen beziehen sich auf Fragen wie: Werden alle Indikationen des Patienten medikamentös abgedeckt? Sind wirklich alle verordneten Arzneimittel indiziert? Ist die Dosierung richtig? Ist die Art der Verabreichung angemessen? Besteht die Gefahr einer Unterdosierung, Überdosierung, Interaktion oder Inkompatibilität? Mit Nutzen/Risiko-Analysen können die klinischen Pharmazeuten einen wesentlichen Beitrag zur Abschätzung und Minderung des arzneimittelbezogenen Risikos leisten [2] und zusätzlich die Medikamentenkosten positiv beeinflussen.
Spitaleintritt und -austritt sind als Schnittstelle zwischen stationärer und ambulanter Betreuung kritische Phasen der medizinischen Behandlung. Die meisten Patienten haben beim Verlassen des Spitals eine andere Arzneimitteltherapie als vor dem Spitaleintritt. Immer häufiger übernehmen klinische Pharmazeuten - in Zusammenarbeit mit dem ärztlichen Team - die medication reconciliation (Medikationsabgleich) bei Eintritt und Austritt sowie das Spital-Austrittgespräch mit dem Patienten. Das Aufzeigen der Unterschiede und das Erklären der neuen Therapie vermindern das Risiko eines plötzlichen Therapieabbruchs oder einer Doppelbehandlung. Das Programm «progress! Sichere Medikation an Schnittstellen» der Patientensicherheit Schweiz hat sich ebenfalls dieser Problematik verschrieben. Eine weitere Massnahme zur Verbesserung der Medikationssicherheit ist die Einführung des elektronischen Patientendossiers [3], dessen gesetzliche Grundlage gegenwärtig in den Räten im Bundesbern diskutiert wird. Das ePatientendossier kann aber nur dann zur Erhöhung der Patientensicherheit beitragen, wenn das Führen eines elektronischen Patientendossiers für Medizinalpersonen, die Medikamente abgeben oder verabreichen, in standardisierter Form obligatorisch ist. Unvollständige Medikationslisten täuschen eine falsche Sicherheit vor - es muss das «Alles-oder-nichtsPrinzip» gelten!

\section{Sicherheit im Umgang mit Hochrisiko-Medikamenten}

Alle injizierbaren Medikamente (Parenteralia) gelten als Hochrisiko-Medikamente. Um die Sicherheit ihrer Anwendung zu verbessern, müssen Risiken erkannt und für spätere Verbesserungsmassnahmen priorisiert werden. Zu diesem Zweck hat die Arbeitsgruppe Qualität und Sicherheit der GSASA das Parenteralia Self Assessment Tool (PSAT) entwickelt. Der Fragebogen erlaubt eine gezielte Evaluation der für die Medikationssicherheit relevanten Aspekte des ParenteraliaHandlings, von der Arzneimittelbeschaffung über die Zubereitung und Verabreichung bis hin zur Schulung des medizinischen Personals. Damit ist es jedem einzelnen Spital möglich, institutions-spezifische Quali- 
tätslücken $\mathrm{zu}$ identifizieren und $\mathrm{zu}$ priorisieren. Ein Protokoll zur standardisierten schweizweiten Anwendung des PSAT gestattet der Arbeitsgruppe Qualität zusätzlich die Identifikation nationaler «Hotspots» für die effiziente Entwicklung evidenzbasierter Verbesserungsmassnahmen. Mit der finanziellen Unterstützung der Firma Biotest werden nationale Daten seit Anfang 2015 strukturiert erfasst und ausgewertet. Eine erste Analyse zeigt, dass das PSAT sich sehr gut eignet, Risiken im Umgang mit Parenteralia zu erfassen. $\mathrm{Zu}$ den bisher identifizierten nationalen Hotspots gehört der Mangel an Sicherheitsbarrieren bei der Anwendung von Parenteralia wie beispielsweise die Definition von Minimal- und Maximaldosierungen sowie die Doppelkontrolle der Infusionspumpen-Programmierung.

\section{Sicherheit der Identifikation der Arznei- mittel - Vermeiden von Verwechslungen}

Gleich aussehende Medikamentenschachteln, unzureichend bedruckte Blisterpackungen und schlecht beschriftete Ampullen führten in der Vergangenheit immer wieder zu Fehldosierungen oder Verabreichung falscher Medikamente. Deshalb spielt heute bei der Auswahl der Medikamente für den Gebrauch im Spital neben therapeutisch-pharmakologischen und ökonomischen Aspekten auch die Sicherheit vor Verwechslungen eine wichtige Rolle.

Eine eindeutige Identifikation jeder einzelnen Arzneimitteldosis vor ihrer Verabreichung trägt massgeblich zur Patientensicherheit bei. Jede Arzneimittelverabreichung sollte am Patientenbett nochmals kontrolliert und validiert werden, am besten elektronisch mittels Barcodes (bedside-scanning) [4]. Neben den technischen Hilfsmitteln setzt dies auch voraus, dass für jede einzelne Arzneidosis die sie umschliessende Primärverpackung (bei Tabletten z.B. Blisterfolie) mit den wichtigsten Daten wie Name, Dosierung, Chargennummer und Verfallsdatum versehen ist. Die Spitalapotheker setzen sich für die flächendeckende Umsetzung dieser mehrwertbringenden Massnahme ein.

Vor drei Jahren hat die GSASA in Zusammenarbeit mit der pharmazeutischen Industrie und Patientensicherheit Schweiz gemeinsame Empfehlungen zur Vermeidung von Verwechslungen wegen ähnlich aussehender Arzneimittel-Verpackungen und-Beschriftungen (Look alike) bzw. zur Vermeidung von Verwechslungen wegen ähnlich klingender Arzneimittelbezeichnungen (Sound alike) verabschiedet. Seither finden diese Empfehlungen selbst bei der Arzneimittelzulassung durch Swissmedic Beachtung.
GSASA - Schweizerischer Verein der Amts-

und Spitalapotheker

1948 gegründet, entwickelte sich der Schweizerische Verein der Amts- und Spitalapotheker (GSASA, ursprünglich Gesellschaft Schweizerischer Amts- und Spitalapotheker genannt) zu einem wichtigen Partner im Schweizer Gesundheitssystem. Neben berufspolitischen Aktivitäten engagiert sich die GSASA sehr stark für Weiter- und Fortbildung, Qualität und Sicherheit sowie für die Weiterentwicklung pharmazeutischer Dienstleistungen im Spital. Durch den eidgenössisch akkreditierten Weiterbildungsgang in Spitalpharmazie und den Fähigkeitsausweis in klinischer Pharmazie können Spitalapotheker ihre Dienstleistungen im komplexen Umfeld des Spitalbetriebs gegenüber Patient und Spital erweitern und verbessern.

Der Vorstand der GSASA ist zuständig für die Sicherstellung der organisatorischen und strategischen Kontinuität des Vereins. Ihm steht eine professionelle Geschäftsstelle für alle administrativen und operativen Aufgaben zur Verfügung. Die GSASA verdankt ihre Aktivitäten aber vor allem mehreren Tausend Arbeitsstunden pro Jahr, die im Milizsystem durch praktizierende Spitalapotheker im Komitee und in diversen Kommissionen und Arbeitsgruppen geleistet werden. Die GSASA hat gut 450 Mitglieder (Stand 2015).

\section{Sicherheit der Arzneimittelversorgung - Lieferengpässe und Marktrückzüge}

Lieferengpässe und Marktrückzüge (letztere insbesondere in der Pädiatrie) nehmen ständig zu. Unterdessen bedarf es einer 40\%-Apothekerstelle pro Spitalapotheke, um die Versorgung der nicht (oder nicht mehr) lieferbaren Medikamente sicherzustellen. Diese meist kurzfristigen Produktumstellungen sind nicht nur logistisch eine Herausforderung. Das Nachführen der Arzneimittelstammdaten im spitalinternen Bestellund Verordnungssystem und die Information des medizinischen Personals über die Produktumstellung sind Folgearbeiten, die vor allem dann kritisch werden, wenn z.B. Produkte mit anderen Wirkstoffen, anderen Wirkstoffkonzentrationen oder Dosierungen substituiert werden müssen. Der Spitalapotheke fällt die Aufgabe zu, den Informationsfluss sicherzustellen und allenfalls Vorsichtsmassnahmen einzuführen. Die GSASA setzt sich seit Jahren dafür ein, dass Lieferengpässe frühzeitig von den Herstellern kommuniziert werden, um die Produktumstellungen besser planen zu können. Leider geben immer noch die meisten Firmen einen Lieferengpass erst bekannt, wenn die Spitalapotheke das Produkt bestellen will und somit nur wenig Zeit bleibt, ein alternatives Produkt zu beschaffen.

Diese Beispiele zeigen, wie insbesondere in den vier Bereichen Verordnung, Anwendung, Identifikation und Versorgung die interdisziplinäre Zusammenarbeit zwischen Spitalapothekern, Ärzten und Pflegefachpersonen mit dem Patienten im Fokus einen wesentlichen Beitrag zur Patientensicherheit leisten kann. 


\section{Referenzen}

1 Bond et al. Clinical pharmacy services, pharmacy staffing, and hospital mortality rates. Pharmacotherapy; 2007.

2 Leape et al. Pharmacists participation on physician rounds and adverse drug events in the ICU. JAMA; 1999.

3 van Doormaal et al. The influence that electronic prescribing has on medication errors and preventable adverse drug events: an interrupted time-series study. J Am Med Inform Assoc; 2009.

4 Poon et al. Effect of bar-code technology on the safety of medication administration. N Engl J Med; 2010.

\section{Weiterführende Links}

- PSAT: www.gsasa.ch/seiten/qualitaet-sicherheit/PSAT/

- Empfehlungen Look alike / Sound alike: http://www.gsasa.ch/ seiten/qualitaet-sicherheit/risikomanagementkonzept-gsasa/

Weiterbildungsgang in Spitalpharmazie: www.gsasa.ch/seiten/ bildung/fph-spitalpharmazie/

- Fähigkeitsausweis in klinischer Pharmazie: www.gsasa.ch/seiten/ bildung/fph-klinische-pharmazie/ 